\title{
MODEL OF BROADBAND METAMATERIAL FOR MICROWAVES
}

\author{
Aleksandr Gorst ${ }^{1}$, Andrei Klokov, Andrei Zapasnoy \\ National Research Tomsk State University, 634050, Tomsk, Russia
}

\begin{abstract}
In this work are presented, results of computer model operation of the structure executed from triangular elements with openings in the center. Results of mathematical calculation of amplitude and phase distribution of an electric field with use of CST Microwave studio are given. Also, calculation of the equivalent index of refraction when passing a plane wave through this structure is given.
\end{abstract}

\section{Introduction}

Metamaterials - the perspective direction of development in various spheres: Development of antenna systems [1], creation of a raincoat of the invisible being [2]. Such materials promote emergence of the new phenomena [3].

All known metamaterials are based on the resonance effect, which includes a resonant circuit. Such metamaterials give a negative value of the refractive index, but this method leads to a sharp decrease in the working frequency band, which is a negative factor. In the 21 st century, with the development of ultra-wideband devices, the use of metamaterials with a $1 \mathrm{GHz}$ band is not appropriate. In turn, scientists suggest the creation of tunable metamaterials, which complicates the structure of the lattice and there is a need to change the layouts from the tasks.

In the previous publications [4, 5] authors showed a possibility of creation of metamaterials from the selfcontained ring conductors focused definitely, placed in polystyrene foam. Creation of such structures processes the long-lived and laborious. The model from ring conductors is rather heavy, its weight is $500 \mathrm{~g}$. The accuracy of manufacturing is not ideal, in view of manual manufacturing. Is there a need for such elements, in the manufacture of which, the human factor would have the least influence, the law minimizing the discrepancy between the parameters of the layout and the model.

\section{Modeling the structure}

The creation of new elements was based on the simplicity of manufacturing and the amplification of the effect of the inverse phase advance in comparison with the model of ring conductors. The structure was based on triangular elements with a hole in the center

\footnotetext{
${ }^{1}$ Corresponding author: gorst93@gmail.com
} 
(Figure 1). The gap between such elements along the axis OY can be considered as a magnetic Hertz dipole, or as a slot antenna and have a significant effect on the magnetic field component. The element itself along its entire length along the OY axis can be considered as a set of electric dipoles with different lengths. Accordingly, this will have an effect on the electrical component of the field. The holes in the triangular elements protrude in the role of the rings, which are combined radiators and interact with both the electrical and magnetic field components, while ensuring the presence of negative magnetization and polarizability. To ensure the repeatability of elements and ease of their manufacture, it is decided to perform them on the basis of printed circuit boards. The same method of fabrication of structures is used by other researchers [6].

Simulation of this structure was carried out using the software CST Microwave studio. The CST Microwave Studio program uses the finite integral method (FIT) - a fairly general approach that first describes the Maxwell equations on a spatial grid, taking into account the energy conservation law, and then by them forms a system of specific differential equations, such as the wave equation or the Poisson equation. The method can be implemented both in the time domain and in the frequency domain. For our case, we use the time domain.

The structure model consisted of 1344 elements (Figure 2). The geometric parameters of the elements are as follows: an equilateral triangle with a side length of $12 \mathrm{~mm}$, a hole radius of $2.5 \mathrm{~mm}$, a triangle distance of $1 \mathrm{~mm}$, and an element thickness of $0.1 \mathrm{~mm}$. The distance between the elements along the $0 \mathrm{X}$ axis is $3 \mathrm{~mm}$, along the $0 \mathrm{Y}$ axis $1 \mathrm{~mm}$, along the $0 \mathrm{Z}$ axis $3 \mathrm{~mm}$. The elements were arranged mirror-like on the dielectric layer $(\varepsilon=3.5)$ with a thickness of $1 \mathrm{~mm}$. The material of the elements is an ideal conductor, the area around the lattice is vacuum, the boundary conditions are open space, the plane wave along the $0 \mathrm{X}$ axis is modeled, the electric field intensity vector is directed along the $0 \mathrm{Y}$ axis, the magnetic field vector is directed along the $0 \mathrm{Z}$ axis in the form of a wide-range pulse signal frequencies of $1-10 \mathrm{GHz}$.

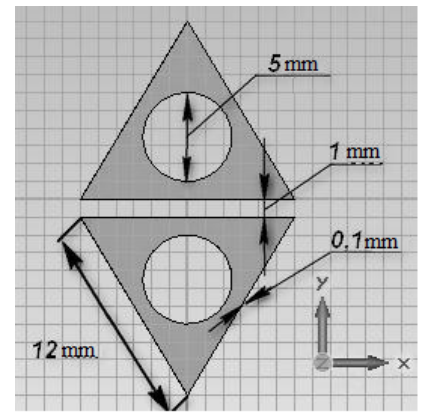

Fig. 1. Model of a triangular element.

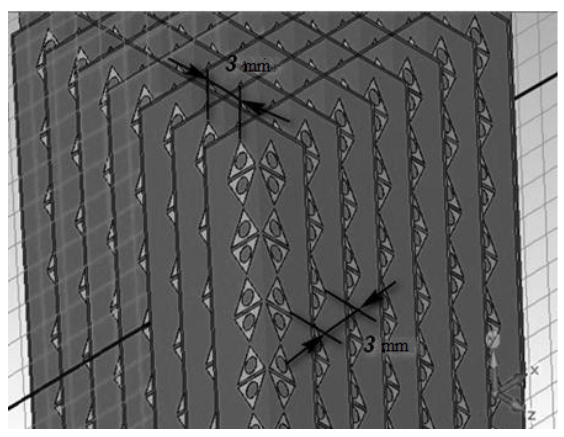

Fig. 2. Lattice model from triangular elements.

Along the black line (Figure 2), the structure passing through the center, necessary data, such as amplitude and phase of the field were taken off. Further for processing data were imported to a mathematical product of Mathcad 14 where necessary calculations were performed.

\section{Simulation results}

In Figure 3 the schedule of phase distribution of the field at a frequency of $4 \mathrm{GHz}$ in the form of dependence of electric distance on distance of field of calculation along the line is submitted. To a layer it is possible to notice oscillation character which is bound to an 
interference of an incident and back wave, as well as in a case with amplitude distribution of the field. In a layer the negative phase attack which demonstrates decrease of an index of refraction is observed. Behind a layer there passes the free wave.

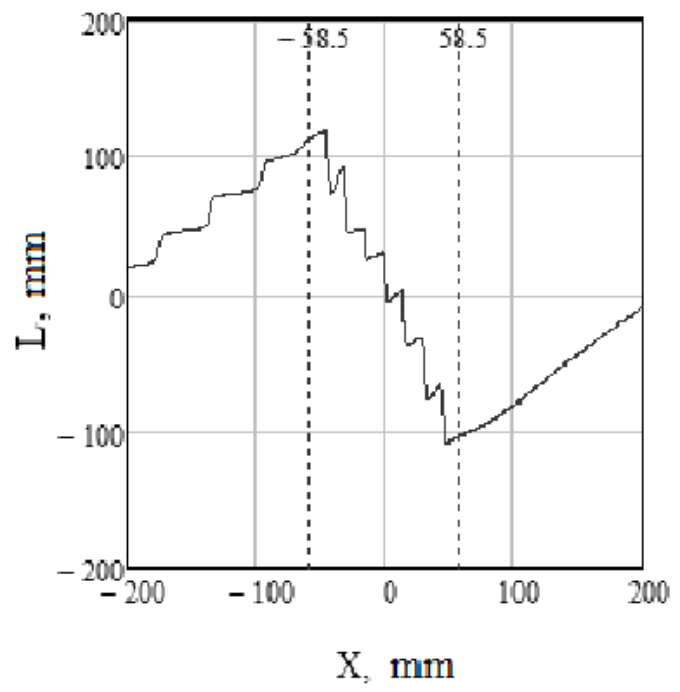

Fig. 3. Phase distribution of an electric field along a straight line at a frequency of $4 \mathrm{GHz}$.

For calculation of a real part of the equivalent index of refraction we use a formula:

$$
n=\frac{\Delta L}{d} \text {. }
$$

where $\Delta L=l_{2}-l_{1}$-wave progression in a layer, $d$ - thickness of structure from triangular elements. For calculation $n$, values $l_{1}$ and $l_{2}$ we take on limits of structure. The real part of the equivalent index of refraction for our structure at a frequency of $4 \mathrm{GHz}$ is equal $n=-2.8$.

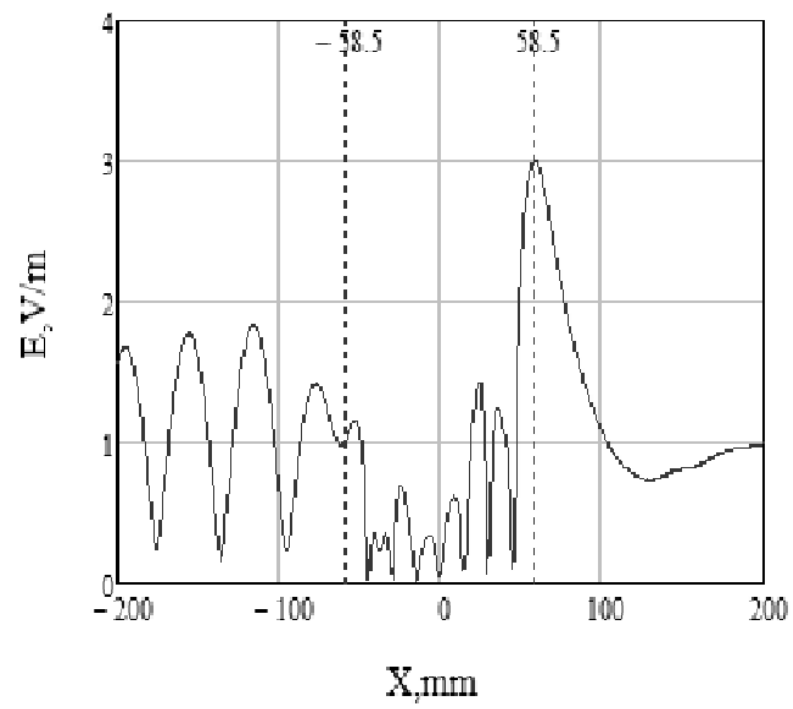

Fig. 4. Amplitude characteristic of a lattice of three layers at a frequency of $4 \mathrm{GHz}$. 
Just pay attention to the distribution of the amplitude of the field E. Figure 4 shows the dependence of the amplitude on the distance, where 0 along the abscissa is the center of the lattice. Markers mark the edges of the model. It can be seen that an interference pattern is observed in front of the layer, which indicates the presence of a reflected wave. The maximum amplitude before the grating is $1.8 \mathrm{~V}$. In the layer, the effect of multiple reflection of waves from the edges is observed, each minimum of the amplitude corresponds to the layer of the lattice. The amplitude above the amplitude of the incident wave whose maximum is equal to $3 \mathrm{~V}$ is observed behind the layer. This effect is due to focusing, which is one of the numerous properties of the material.

The negative value turned out not only on $4 \mathrm{GHz}$, in Figure 5 the schedule of a real part of the equivalent index of refraction for a frequency band $3 \div 7$ of $\mathrm{GHz}$ is given below.

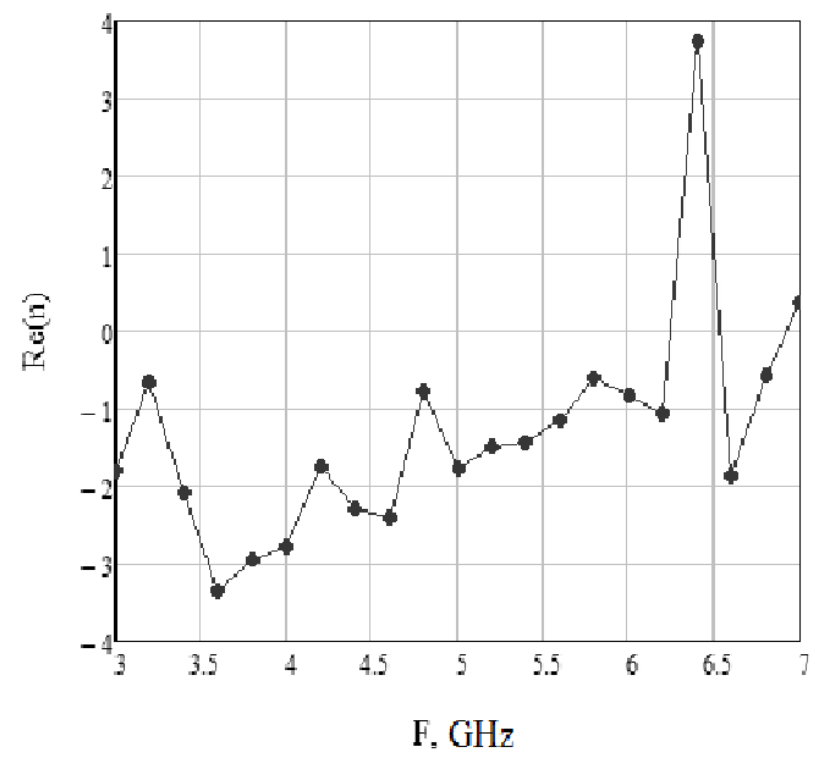

Fig. 5. Dependence of a real part of the equivalent index of refraction on frequency.

Points on graphics noted calculated values of the equivalent index of refraction. Calculation was carried out with a step to $0.2 \mathrm{GHz}$. The minimum value of the equivalent index of refraction is -3.4 . At a frequency of $3.6 \mathrm{GHz}$. Apparently the schedule has the cutup increasing character, but values are negative for a frequency band $3 \div 6.2 \mathrm{of} \mathrm{GHz}$. At a frequency of $6.4 \mathrm{GHz}$ there is a sharp increase, a real part of the equivalent index of refraction, to value $n=-3.7$ further we see just the same sharp decrease. Presumably it is bound to a resonance on which elements interact in such a way that the layer becomes a dielectric.

\section{Conclusion}

Analyzing the obtained data it can be stated that the structure of triangular elements with a cutout in the center, similar to the structure of ring conductors, according to the values of the real part of the equivalent refractive index, but works in a larger frequency range. This structure allows you to get rid of resonance effects with small changes in the value of the refractive index. From the amplitude distribution graph it can be concluded that a certain focusing zone forms behind the layer. 


\section{References}

[1] V. Slyusar, Electronics 3, 44(2010)

[2] W. Cai, U. Chettiar, A. Kildishev, and V. Shalaev, Nat Photon 1, 224(2007)

[3] Alù and N. Engheta, Phys. Rev. 78, 1(2008)

[4] A. Klokov, V. Yakubov, S. Shipilov, A. Gorst A. Mironchev, Electronic means and control systems: Proceedings of the $\mathrm{X}$ International Scientific and Practical Conference 1, 146 (2014)

[5] V. Yakubov, A. Andretsov, I. Ponomareva, A. Mironchev, News of Higher Educational Institutions Physics 53, 17(2010)

[6] J. Pendry, A. Holden, D. Robbins, W. Stewart, IEEE Trans. Microwave Theory Tech. 47, 2075 (1999) 